\title{
Effect of Persian Manual Therapy (Fateh Technique) on Patients with Mild and Moderate Carpal Tunnel Syndrome: A Clinical Trial
}

\author{
Hamed Naeiji', Roshanak Mokaberinejad ${ }^{1 *}$, Seyed Ahmad Raeissadat², Farshad Nouri³, Yousef Fallah4, Reza Haj \\ Manouchehri ${ }^{5}$, Alireza Abbassian6, Seyed Mohammad Riahi
}

\author{
'Department of Traditional Medicine, School of Traditional Medicine, Shahid Beheshti University of Medical Sciences, Tehran, Iran. \\ Physical Medicine and Rehabilitation Department, Clinical Development Center of Shahid Modarres Hospital, Shahid Beheshti University of Medical Sciences, Tehran, Iran. \\ 3Physical Medicine and Rehabilitation Department, School of Medicine, Akhtar Hospiatl, Shahid Beheshti University of Medical Sciences, Tehran, Iran. \\ ${ }^{4}$ Department of Orthopedic Surgery, Joint Reconstruction Research Center, Shariati Hospital, Tehran University of Medical Sciences, Tehran, IR Iran. \\ Legal Medicine Research Center, Legal Medicine Organization, Tehran, Iran. \\ ${ }^{6}$ Department of Persian Medicine, School of Persian Medicine, Tehran University of Medical Sciences, Tehran, Iran. \\ ${ }^{7}$ Cardiovascular Diseases Research Center, Department of Epidemiology and Biostatistics, School of Medicine, Birjand University of Medical Sciences, Birjand, Iran. \\ *Correspondence to: Roshanak Mokaberinejad (E-mail: rmokaberi@gmail.com) \\ (Submitted: 23 March 2021 - Revised version received: 02 April 2021 - Accepted: 25 April 2021 - Published online: 26 August 2021)
}

\begin{abstract}
Objectives Various non-surgical treatments are used to treat Carpal tunnel syndrome, including hand therapy. In this study, the effect of Fateh Iranian hand therapy on this disease has been investigated for the first time.

Methods In this controlled clinical trial, 58 female patients (78 hands) eligible for carpal tunnel syndrome were divided into two groups of intervention (splint, Fateh hand therapy, and exercise) and the control group (splint only). Each person in the intervention group received about 7 minutes of soft tissue manipulation for 6 sessions and performed two active exercises at home daily. Symptom severity and functional capacity were assessed with the Boston questionnaire, pain intensity, and electrodiagnostic findings at the beginning and tenth week, and patient satisfaction in the tenth week of the experiment.

Results Data of 51 patients, all female (68 hands), were analyzed. The age distribution was the same in both groups. In the intervention group, we saw a significant improvement in symptom severity and functional capacity compared to the control group $(P$-value $<0.05)$. In addition, pain changes in the intervention group were significantly more than in the control group ( $P$-value $<0.05)$. The values of electrodiagnostic variables at the beginning and end of the design were not significantly different between the two groups $(P$-value $>0.05)$. Comparison of changes in these values did not show a significant difference between the two groups $(P$-value $>0.05)$. Satisfaction in the intervention group was significantly higher ( $P$-value $<0.05$ ).

Conclusion Fateh method is effective in reducing the symptoms of mild to moderate carpal tunnel syndrome.

Keywords Carpal tunnel syndrome, traditional medicine, complementary therapies, Persian manual therapy, radpa
\end{abstract}

\section{Introduction}

Carpal Tunnel Syndrome (CTS) is a common, chronic, and debilitating disorder. This disease is the most common peripheral mononeuropathy that occurs mainly in adults of working age $^{1}$ and is one of the most common causes of activity limitation in these people ${ }^{2}$ and often occurs in patients aged 30 to 60 years. ${ }^{3}$ It affects most women and increases with age (Agnessa et al.). ${ }^{4}$ Carpal tunnel release is one of the most common hand/ wrist procedures, ${ }^{5}$ with more than 400,000 carpal tunnel release surgeries performed each year, with a direct cost of more than $\$ 2$ billion per year. ${ }^{6}$ The prevalence of this disease varies depending on the diagnostic criteria and is estimated from $4-5 \%{ }^{7}$ to $7-19 \%{ }^{1}$ Its prevalence in women is two to three times higher than men. ${ }^{3}$ The annual incidence of this disease has been 99 per 100 thousand. ${ }^{1}$ Although carpal tunnel syndrome is mostly idiopathic, various diseases such as hypothyroidism, diabetes, rheumatoid arthritis, chronic renal failure, acromegaly, amyloidosis, hemophilia, gout, fractures, and trauma to the carpal tunnel, and obesity, pregnancy, and mitochondrial disease can accelerate its progress. Carpal tunnel syndrome ultimately leads to surgery when progresses and in the lack of supportive therapies. ${ }^{8}$

Therapies include surgical and non-surgical treatments. The non-surgical treatments used for this condition include a variety of options, including changing habits such as restricting the movement of the wrist joint and reducing strenuous occupational activity; using ergonomic devices, using splints or a variety of oral medications such as oral steroid medications as well as ultrasound therapy, ${ }^{9}$ injection of steroid medications, ${ }^{10}$ taking nonsteroidal anti-inflammatory drugs (NSAIDs), Diuretics, Vitamin B6, Job change, ${ }^{11,12}$ topical injection of progesterone, ${ }^{13,14}$ Botulinum toxin injection, Use of magnets, ${ }^{15}$ Iontophoresis, Low-level laser, ${ }^{16}$ Median nerve mobilization, ${ }^{17}$ Scaphoid and Hamate Mobilization, ${ }^{18}$ Static magnetic field (SMF), Bioptron, ${ }^{19}$ specific exercise, ${ }^{20}$ Plateletrich plasma $(\mathrm{PRP})^{21}$ and ozone therapy ${ }^{22}$ are other suggested non-surgical treatments.

Hand therapy is one of the non-surgical conservative treatments for carpal tunnel syndrome. Although there are various results of the effectiveness of manual therapy in the treatment of carpal tunnel syndrome, which varies from ineffective to useful, there is evidence to suggest that different methods of manual therapy of the wrist area are effective in the treatment of carpal tunnel syndrome and can help reduce pain and symptoms. ${ }^{18,23,24}$

In this study, we introduce an Iranian manual therapy method called Fateh hand method, which is one of the manual therapies of Iranian folklore. About four decades ago, Mohammad Fateh invented manual therapy methods to manage lower and upper limb problems. This study introduces upper limb manual therapy of this method. Later, his son Ahmad 
Fateh modified these methods and combined them with two active exercises at home. ${ }^{25}$ The speed and ease of treatment, no need for routine massage preparations, easy learning and movements by patients, the limited number of treatment sessions, and very rare side effects made this manual therapy for the first time in the form of a clinical trial that we investigated.

In this study, the therapeutic effects of this method along with the use of splints have been compared with a group that uses only splints (as a treatment method whose beneficial effects on CTS have been confirmed in many studies). To evaluate the effects of this method, we used the analysis of its effects on the severity of symptoms and functional capacity of the hand, the amount of pain as well as electrodiagnostic parameters.

\section{Materials and Methods}

\section{Study Design}

It was a multicenter clinical trial study with a parallel-group design. This study was performed from 2019 to 2020 in Sina and Shahid Modarres hospitals in Tehran, Iran. 58 patients (78 hands) with CTS who referred to neurology, orthopedics, physical medicine and rehabilitation or traditional medicine clinics were included in this study. Participants were randomly divided into two groups. The intervention group was designated as "splint + hand therapy + exercise" and the control group was designated as "splint group".

Although it was impossible to blind the participants due to dissimilarity of the interventions in the two groups, the process of preparing the nerve tape, filling out the questionnaire, and the data analyzer were blinded.

\section{Ethical Considerations}

This study was approved by the Research Ethics Committee of the Vice Chancellor for Research and Technology of Shahid Beheshti University of Medical Sciences on November 4, 2018 (reference number: IR.SBMU.RETECH.REC.1397.667). All stages of the study were conducted in accordance with the Helsinki Declaration of Human Rights. The registration number of the clinical trial is IRCT20190429043421N1.

All patients were informed about the study and its processes before entering the study, and while receiving informed written consent from all participants, they were told that if they did not wish, they could leave it at any stage of the study. Confidentiality of patients' identity and personal information has been observed.

\section{Participants}

The participants were women aged 20 to 60 years who were included in the study after confirming their affliction with carpal tunnel syndrome by a physician. In terms of inclusion and exclusion criteria, 58 patients (78 hands) entered the study, which were divided into two groups of intervention (splint + manual therapy + exercise) and control group (splint). During the study, 4 people (6 hands) of the first group and 3 people (4 hands) of the second group were excluded from the study due to lack of complete referral for manual therapy or incomplete follow-up sessions, and finally, two groups with 24 people ( 34 hands) remained in the first group and 27 people (34 hands) in the second group and the study was performed on them (Figure 1).

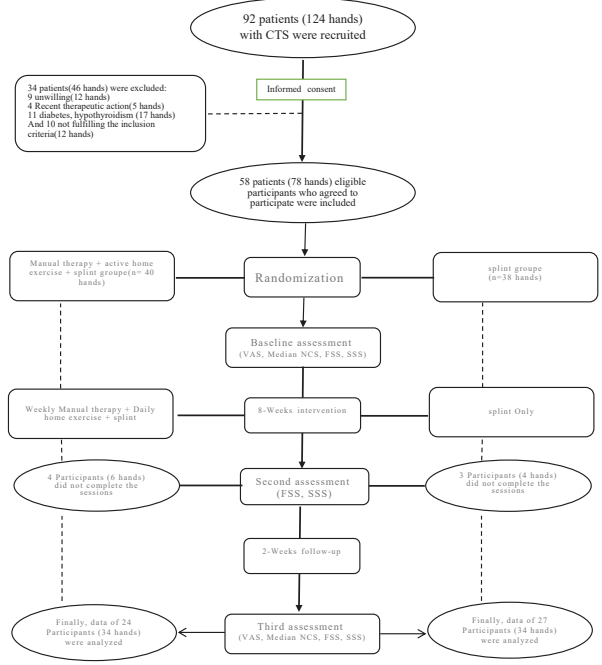

Fig. 1 Flow diagram of phases through clinical trial.

\section{Clinical Diagnosis Criteria}

Having at least two signs, or a sign and a symbol of the following:

Symptoms include nocturnal pain, paresthesia, numbness, and tingling in the hands, and symptoms include a positive phalanx or tunnel test.

Criteria for diagnosing electrodiagnosis by mild to moderate type:

Sensory peak latency longer than 3.6 milliseconds with normal motor onset latency ( $\leq 4.2$ milliseconds), mild carpal tunnel syndrome, and sensory peak latency longer than 3.6 milliseconds with prolonged motor onset latency (4.3-6 milliseconds) is considered moderate carpal tunnel syndrome.

\section{Study Inclusion Criteria}

Women in the age range of 20 to 60 years and the clinical diagnosis of their disease are proposed by a specialist and confirmed by electrodiagnosis, mild to moderate type and at least 3 months have passed since the onset of their symptoms if there are no exclusion criteria, then By entering the informed consent form and accepting the conditions of participation in the research, they enter the project.

\section{Study Exclusion Criteria}

A history of wrist trauma or surgery, a history of carpal tunnel syndrome treatment in the last three months, including physiotherapy, laser therapy, or any injections such as steroids, pregnancy at the time of enrollment, a history of diabetes based on the patient's diagnosis, or based on clinical suspicion and tests during the study and thyroid disorder at the discretion of the patient, median nerve involvement in areas other than the carpal tunnel area that is confirmed by a clinical diagnosis and NCV, presence of cervical radiculopathy, history of collagen vascular diseases such as arthritis Rheumatoid arthritis, scleroderma, amyloidosis, lupus, concomitant with other known tenosynovitis approved by the rheumatologist, advanced cancer or recent chemotherapy and radiotherapy, anticancer drugs, corticosteroids, immunosuppressive drugs and sedatives severe medical conditions such as kidney and heart failure, obesity or excessive weight loss (based on BMI), 
cases outside the range of mild to moderate disease (mentioned in the inclusion criteria) with symptoms such as tendon atrophy and permanent paresthesia outside the range of mild disease to medium (mentioned in the inclusion criteria) with symptoms such as tendon atrophy and permanent paresthesia.

\section{Assessment of patients}

\section{Boston Questionnaire}

Symptom Severity and Functional Status were evaluated using the Boston Carpal Tunnel Syndrome Questionnaire (BCTQ). The questionnaire consists of 2 separate sections: Symptom Severity Scale (SSS) with 11 questions about severity and frequency of symptoms such as night and daily numbness, causalgia and pain and Functional Status Scale (FSS) with 8 questions about some specific daily activities such as writing, close the dress button, holding the phone and ...; each question has 5 options and each option has a score of 1 to 5 , in which 1 indicates the lack of symptoms and 5 indicates the most severe symptoms. Patients with bilateral CTS completed separate BCTQ for each hand. All patients completed BCTQ at first, the fourth, and the tenth weeks. The studies conducted indicate the reliability and validity of the translated version of this questionnaire in a valid Persian in assessing the severity of symptoms and the function of carpal tunnel syndrome in Iranian society. ${ }^{26,27}$

\section{Electrodiagnosis}

Signs and symptoms of carpal tunnel syndrome were investigated in all patients and after the clinical approval of the diagnosis of carpal tunnel syndrome, to confirm the diagnosis, electrodiagnostic evaluation of the patients was performed by physical medicine and rehabilitation specialists or neurologists. Nerve conduction studies were prescribed by the treating physician and performed in an electromyography laboratory by experienced personnel.

\section{Pain Evaluation}

The pain level in patients' hands and fingers was questioned during the week before referral and evaluated on a numerical rating scale (VAS). On this scale, "zero" indicates painlessness, and 10 indicates unbearable pain. In patients with bilateral CTS, pain in each hand was calculated separately. Pain levels were assessed at baseline and at the 10th weeks.

\section{Evaluation of Satisfaction}

The rate of patient satisfaction is reflected from treatment on a 5-degree scale of 1-5. On this scale 1 is considered as very dissatisfied and 5 as very satisfied.

\section{How to Intervene}

After completing a standard questionnaire for Symptom Severity and Functional Status of the patient's hand (BCTQ), patients were divided into two groups. Splint was prescribed for participants in both groups. The splint was prescribed in a notary mode (0-5 degree of extension) for 8 weeks of complete nights and days in most hours of activity. Manual therapy was also performed weekly (up to 4 weeks) and 2 sessions in weeks 6 and 8 (total 6 sessions) for patients in the intervention group and daily exercises was also taught to them to be performed at home 3 times per day and for 8 weeks. Patients with more than 2 sessions absence were excluded from the study. To measure the severity of the symptoms of the disease, for each patient, in addition to the beginning of the plan, in week 4, the Boston questionnaire was completed again. In the tenth week, in addition of completing the Boston questionnaire, the amount of pain and treatment satisfaction and nerve strip was also investigated.

\section{Manual Therapy Method}

The first part of the manual therapy includes shoulder fraction and pressure insertion at three points, and especially the middle point of the shoulder with high pressure, rotational and fast rhythm with about 2 minutes (Figures 2).

Then, the deep petrissage in the arm front splint with the index finger, moving from the bottom to the top and fast and repeated with an approximate time of 20 seconds.

High pressure, fast and rhythmic rotational friction of the scapula inner sides with the thumb (Fig. 1), reaching the lower end of the scapula with a deep transverse petrissage movement from inside (medial) to outside (lateral at the base of the scapula with the thumb, which takes about 30 seconds).

The main part of this manual therapy is done in its second part. At this stage, for manual treatment of the upper right limb, while the patient leans to the chair, the therapist should stand on the right side of the patient and hold the patient's wrist with the left hand, while the angle between the patient's forearm and arm is about 90 degrees. Then the therapist raises the arm to the shoulder circumference and about 3-4 fingers below the armpit deep petrissage with the fingers of the right hand from outside to inside, with high pressure, fast rhythm, and repetitive movements will be performed. This step takes about $10-15$ seconds (Figures $3 \mathrm{~A}$ and $3 \mathrm{~B}$ ).

Forearm petrissage is performed at four to five points with moderate pressure, fast rhythm and once at each point along the forearm for 10 seconds.

After this step, friction is performed on the back of the hand in the area between the index finger and the thumb. Friction is performed with relatively high pressure, medium rhythm and between one to three times and each time with a time of 10 seconds (Figure 4).

\section{Exercises at home}

The intervention group participants were asked to perform daily exercises at home. Exercises were described to the participants, along with images shown exercises performance, tables were given to record daily exercises. In each stage, patients

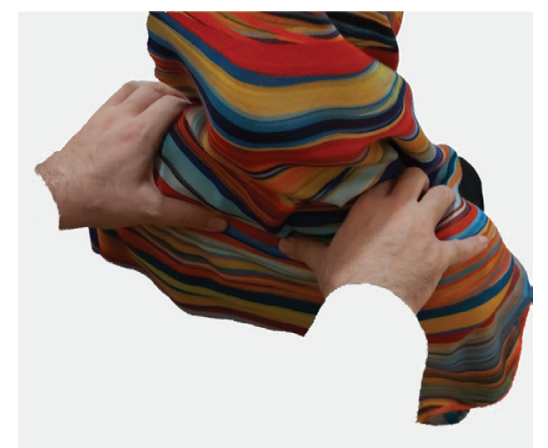

Fig. 2 Rotational friction of the inner sides of the scapula with the thumbs. 

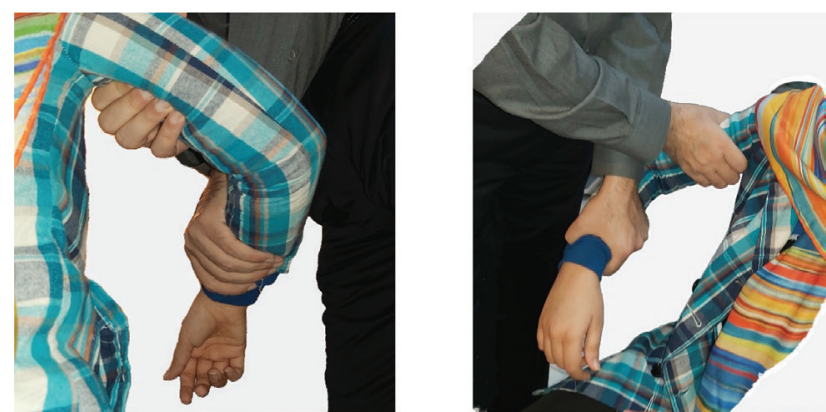

Fig. 3 (A, B) Deep petrissage of the below armpit.

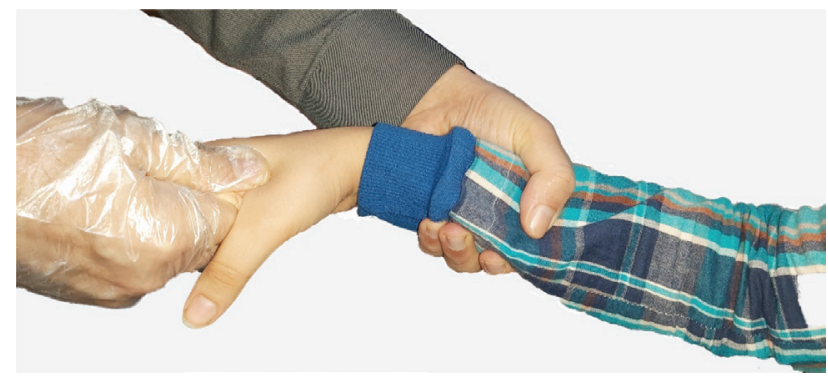

Fig. 4 Friction of the area between the index finger and thumb.

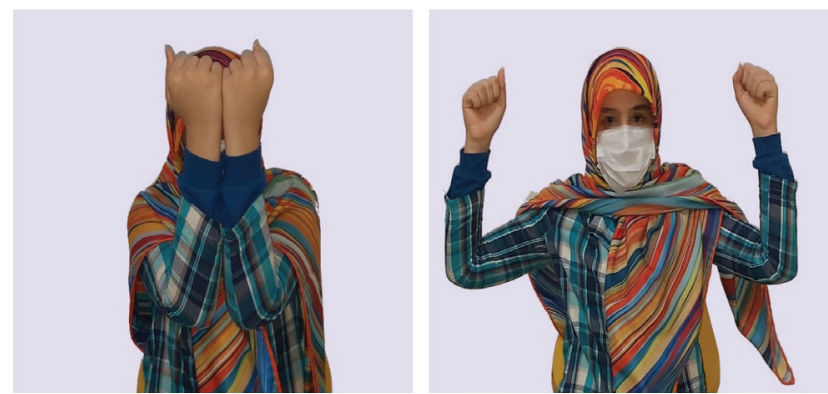

Fig. 5 (A, B) Exercise at home 1.

referring to manual therapy, training tables were examined to check the exercise rate. Each exercise should be performed daily 3 times and each 10 time (for 6 weeks).

In the first exercise, the patient bends his forearm from the elbow to 90 degrees and punches and holds the hand in front of the face, then with a horizontal movement outwards, places it around the shoulders and returns the hands to the first position. The movements are performed continuously and at a medium speed. The patient performs this movement 10 times (Figures 5A and 5B).

In the second exercise, the patient is asked to and bring the hands in front the body while ring hands, bring them up to the top of the head, and return it to its original position, performing this movement 10 times (Figures 6A and 6B).

\section{Statistical Analysis}

In describing data according to the variable type, for quantitative values, the mean index and standard deviation were used, and we used the frequency and percentages for qualitative variables. Shapiro-Wilk test was used to evaluate the normality of the data. In order to examine single-variable relations in the two groups of intervention and control, nonparametric tests (Wilcoxon \& Mann-Whitney Test) were used to compare the independent and dependent samples. Chi-square test was used to compare qualitative variables in the two groups. A significant level in this study is 0.05 . The analyzes were performed using IBM SPSS Statistics V.25 software.

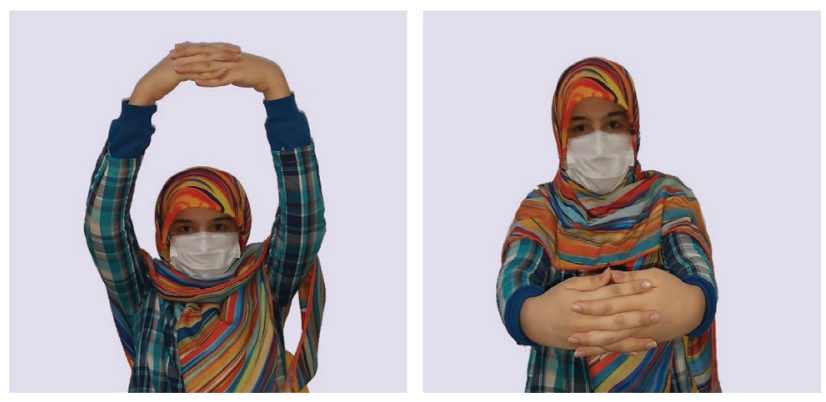

Fig. 6 (A, B) Exercise at home 2.

\section{Results}

\section{Demographic Data}

The data of 58 ( 78 hands) that were included in the study were analyzed. Data from 7 people (10 hands) were excluded from the study in different stages because participants performed less than $80 \%$ of daily exercises or did not participate in the minimum designated manual therapy sessions. 4 (6 hands) of these cases were related to the intervention group and 3 (4 hands) were related to the control group. Finally, 51 patients (68-handed) were analyzed in two groups, of which 34 hands (24 people) were related to the intervention group and 34 hands (27 people) were related to the control group (Chart 1 ). It should be noted that in this study in a complete 6 session period, from 24 patients in the intervention group, 11 people in 4 sessions of manual therapy (2 sessions of absence), and 8 people in 5 sessions of manual therapy (1 session of absence) participated, and 5 people completed the 6-session course, participants were excluded from the study with more than 2 sessions of absence.

All participants in the study were female. The mean age of participants in the two groups did not differ significantly $(P$-value $>$ $0.05)$. In the intervention group, 10 were employed and 14 housewives and in the control group, 11 were employed and 16 housewives. In the study of dominant hand and involved hand in both groups, there was a significant relationship between involved hand and dominant hand $(P$-value $<0.05)$ (Table 1$)$.

\begin{tabular}{|c|c|c|c|c|}
\hline & $\begin{array}{l}\text { Intervention } \\
\text { group }\end{array}$ & Control group & Total & $P$-value \\
\hline Women, $n$ & 24 (34 hands) & 27 (34 hands) & 51 & \\
\hline $\begin{array}{l}\text { Age (Mean } \pm \text { SD), } \\
\text { year }\end{array}$ & $49 \pm 6$ & $49 \pm 7$ & & $0.721^{a}$ \\
\hline \multicolumn{5}{|l|}{ Employment } \\
\hline Occupy & $10(41.7 \%)$ & $11(40.7 \%)$ & 21 & $0.948^{a}$ \\
\hline Housewife & $14(58.3 \%)$ & $16(59.3 \%)$ & 30 & \\
\hline \multicolumn{5}{|l|}{ Symptomatic hand } \\
\hline Right, n (\%) & $10(41.7 \%)$ & $9(33.3 \%)$ & 19 & $0.486^{a}$ \\
\hline Left, $n(\%)$ & $4(16.6 \%)$ & $11(40.7 \%)$ & 15 & \\
\hline Both, $n(\%)$ & $10(41.7 \%)$ & $7(25.9 \%)$ & 17 & \\
\hline \multicolumn{5}{|l|}{ Dominant hand } \\
\hline Right, $n$ (\%) & $19(79.2 \%)$ & $18(66.7 \%)$ & 37 & $0.574^{a}$ \\
\hline Left, $n(\%)$ & $5(20.8 \%)$ & $9(33.3 \%)$ & 14 & \\
\hline
\end{tabular}

astudent $t$ test. 
Table 2. Intragroup and intergroup comparison of average functional capacity Scale, Symptom Severity Scale, and Pain in two groups of intervention and control

\begin{tabular}{|c|c|c|c|c|c|c|c|c|}
\hline & & Baseline & 10 weeks later & ${ }^{\dagger} \boldsymbol{P}$-value & $\begin{array}{l}\text { Changes } \\
\text { (Base-10) }\end{array}$ & ${ }^{t+P}$-value & $\begin{array}{l}{ }^{t+P} \boldsymbol{P} \text {-value } \\
\text { Baseline }\end{array}$ & $\begin{array}{c}{ }_{t+t t} \boldsymbol{P} \text {-value } \\
10 \text { w. I. }\end{array}$ \\
\hline \multirow{2}{*}{ BCTQ-SSS } & Control & $28.6 \pm 8$ & $18.3 \pm 7$ & $<0.001^{a}$ & $10.2 \pm 5.1$ & \multirow{2}{*}{$0.003^{\mathrm{a}}$} & \multirow{2}{*}{$0.012^{\mathrm{a}}$} & \multirow{2}{*}{$0.658^{a}$} \\
\hline & Intervention & $34.6 \pm 10$ & $18.3 \pm 6$ & $<0.001^{a}$ & $16.2 \pm 8$ & & & \\
\hline \multirow{2}{*}{ BCTQ-FSS } & Control & $17.5 \pm 6$ & $12 \pm 4$ & $<0.001^{a}$ & $5.12 \pm 4.3$ & \multirow{2}{*}{$0.027^{\mathrm{a}}$} & \multirow{2}{*}{$0.028^{a}$} & \multirow{2}{*}{$0.120^{\mathrm{a}}$} \\
\hline & Intervention & $22.8 \pm 10$ & $14.1 \pm 5$ & $<0.001^{a}$ & $9.7 \pm 5.9$ & & & \\
\hline \multirow{2}{*}{ Pain } & Control & $5.2 \pm 2.1$ & $2.3 \pm 1.5$ & $<0.001^{\mathrm{a}}$ & $2.9 \pm 1$ & \multirow{2}{*}{$0.006^{a}$} & \multirow{2}{*}{$0.003^{\mathrm{a}}$} & \multirow{2}{*}{$0.728^{a}$} \\
\hline & Intervention & $6.8 \pm 2.9$ & $2.6 \pm 2.1$ & $<0.001^{\mathrm{a}}$ & $4.2 \pm 2$ & & & \\
\hline
\end{tabular}

BCTQ, Boston Carpal Tunnel Questionnaire; SSS, Symptom Severity Scale; FSS, Functional Status Scale. 'Intragroup comparison of base values and tenth weeks, ${ }^{++}$Comparison of average changes between two groups, ${ }^{+t+}$ - Intergroup Basic values comparison, ${ }^{+t+\dagger}$ Intergroup comparison of tenth week values ${ }^{\mathrm{a}}$ Mann-Whitney $U$ test.

\section{Symptom Severity and Functional Status}

Symptom Severity and Functional Status were evaluated using the Boston Carpal Tunnel Syndrome Questionnaire (BCTQ). Comparison of mean values of Symptom Severity Scale and Functional Status Scale were significantly different between the two groups $(P$-value $=0.012)$. The intragroup comparison of the changes in these two items during the study period in the two groups indicated improved symptoms in both groups.

Furthermore, comparing the changes in these two items between the two groups showed more improvement in both Symptom Severity and Functional Status items in the intervention group, which was significant compared to the control group $(P$-value $<0.005)$ (Table 2$)$. This means that the performed intervention had an additional effectiveness, in terms of decreasing the symptom severity and improving the functional status, compared to the control group (splint only).

\section{Pain}

To compare pain variables, the first changes in pain score based on a visual scale (VAS) in each group were investigated separately. The mean pain at the beginning of the study was higher in the intervention group than the control group, which after ten weeks, the pain intensity decreased in both groups, but this reduction was significantly greater in the manual therapy group (Table 2).

\section{Electrodiagnostic Tests}

In this experiment, Sensory Peak Latency and Motor Onset Latency are measured as the basis of evaluation. Accordingly, the baseline values of Sensory Peak Latency and Motor onset latency were compared between the two groups, which did not differ significantly ( $P$-value $>0.05)$.

In addition, the values of Sensory Peak Latency and Motor onset latency were also measured in the tenth week of entry of patients to the study, which did not differ significantly ( $P$-value $>0.05)$, and this means that the effectiveness of manual therapy in the intervention group did not differ significantly with the control group (Table 3 ).

There was a significant difference between the two groups in terms of disease severity and number of mild and moderate cases at the beginning of treatment $(P$-value $=0.006)$, while after ten weeks from the beginning of the intervention, this difference was not significant $(P$-value $=0.086)$.

\section{Satisfaction}

The patients were asked in the tenth week to determine the rate of satisfaction, which were $6.9 \pm 1.3$ and $7.5 \pm 0.8$ in the control and intervention groups, respectively. Data analysis showed that there was a significant difference between the two groups $(P$-value $=0.046)$.

\section{Discussion}

Fateh manual therapy is one of the manual therapies used in traditional Iranian medicine $\left(\mathrm{RADPA}^{1}\right)$. In the present study, the effect of Fateh manual therapy intervention on the symptoms of carpal tunnel syndrome was investigated and showed that this treatment could be effective in improving Symptom Severity, Functional Status, and reduced pain of the patients who were investigated using VAS and BCTQ and the electrodiagnostic parameters. Therefore, the use of this treatment along with other common treatments, including the use of a splint, can bring better treatment results for the patient.

In the Fateh manual therapy, soft tissue manipulation to relax the muscle, improve circulation, stimulate tendons and nerves, and pain modification are used (Sanei et al., 2020). ${ }^{28}$ In this technique, contrary to many manual therapies where the wrist is the location of manipulation (Elliott and Burkett, 2013; Madenci et al., 2012), ${ }^{12,29}$ manipulation in other areas of the upper limbs, especially the under the armpit area, is somehow distinguished from the usual methods.

This technique consists of two sections, especially in the second part, the main manual therapy of soft tissue is used. In traditional Iranian medicine, this practice is called Ghamz, which means severe pressure on the tissue using the fingers. ${ }^{30,31}$ In this type of treatment (Ghamz), communication channels are introduced that show the movement of substances in the body. "Ghamz Therapy" facilitates the transmission of these substances and reduces the severity of diseases by absorbing substances, diverting harmful currents and improving blood circulation in the affected organ.

So far, there is no study on this manual method and its therapeutic effects on carpal tunnel syndrome, and this study is the first research in this area. There are several points in this treatment that distinguishes it from other manual therapy practices, one of which is the performance speed, while the duration of this treatment is about 7 minutes, many 'Rahkarha-ye Darman-e Dasti-ye Parsi 
Table 3. Comparison of electrodiagnostic findings in two groups of intervention and control

\begin{tabular}{|c|c|c|c|c|c|c|c|}
\hline & & \multicolumn{2}{|c|}{ Baseline } & \multicolumn{2}{|c|}{10 weeks later } & \multicolumn{2}{|c|}{${ }^{\dagger} \boldsymbol{P}$-value } \\
\hline & & Control g. & Intervention g. & Control g. & Intervention $\mathbf{g}$. & Baseline & $\begin{array}{c}\text { Value } 10 \\
\text { weeks later }\end{array}$ \\
\hline \multicolumn{2}{|c|}{ Sensory peak latency } & $0.6 \pm 4.1$ & $0.6 \pm 4.1$ & $0.6 \pm 3.9$ & $0.6 \pm 3.9$ & $0.853^{\mathrm{a}}$ & $0.772^{\mathrm{a}}$ \\
\hline \multicolumn{2}{|c|}{ Motor onset latency } & $0.6 \pm 4.1$ & $0.7 \pm 4.2$ & $0.6 \pm 4.0$ & $0.6 \pm 4.0$ & $0.152^{a}$ & $0.555^{a}$ \\
\hline \multirow{2}{*}{ Severity } & Mild & 25 & 22 & 26 & 24 & \multirow{2}{*}{$0.006^{b}$} & \multirow{2}{*}{$0.086^{b}$} \\
\hline & Moderate & 9 & 12 & 8 & 10 & & \\
\hline
\end{tabular}

${ }^{\mathrm{a}}$ Mann-Whitney $\mathrm{U}$ test. ${ }^{\mathrm{b}} \mathrm{Chi}$-Square test. ${ }^{\dagger}$ Intergroup comparison of base values and tenth weeks.

conventional massages mostly need more times. ${ }^{29,32,33}$ Meanwhile, the number of massage sessions in accordance with the protocol is a weekly session, and a total of 6 sessions.

The next point is the ease of doing so that it can be done on clothes and does not need special peripheral readiness and special readiness for the patient.

The third point is related to manipulation location. In this technique, unlike many common massages - as described in manual therapy - the wrist that is considered as the main location involved in the physiopathology of this disease is not manipulated. ${ }^{12,23,29,32}$

For the study and in order to comply with ethics in research and not depriving patients from common and evaluated treatments, splint was used in both groups. For the first time in 1947, the splint was used in a study by ROAF in carpal tunnel syndrome. ${ }^{12}$ In the present study, the Fateh manual therapy was compared with the splint treatment that is fully characterized by validity and reliability to evaluate its efficiency and reliability. Therefore, we compared the results of the intervention group (manual treatment with splint) with the control group that used splint merely.

In previous studies, different manual interventions (based on soft tissue, carpal bone treatment, and median nerve and mobilization) showed signs reduction in patients with carpal tunnel syndrome, ${ }^{12,34,35}$ which in some survival studies of these signs were also evaluated and approved for months. ${ }^{24}$ In the study of Linek \& Wolny, which was performed on 103 patients with mild and moderate CTS Symptom Severity and Functional Status, pain significantly decreased, ${ }^{36}$ which is consistent with the findings of our study. We also saw similar results in another study of Wolny et al..$^{23}$ as well as Madenci et al. ${ }^{12}$

The nerve conductivity velocity is not evaluated in all studies, and in most of them, statistical evaluation has not been performed due to the low number of recorded patients, and additionally, considering the difference in manual therapy and their prescribed time, definitive conclusions in the effect of these interventions have not been performed in neurophysiological registration. ${ }^{35}$ But in the Wolny et al. study, conducted on the effectiveness of manual therapy in the treatment of carpal tunnel syndrome, we have seen a significant improvement in the Sensory Conduct Velocity and Motor Conductive Velocity in the manual therapy group and reduced Motor Latency in both groups. ${ }^{23}$ In the Madenci et al. study, in the intervention group (massage associated with a splint) there was a significant decrease in $\mathrm{mMDL}^{2}$ levels after treatment compared to pre-treatment values, however, the difference between these values with the control group (splint) was not significant. ${ }^{12}$ In our study, changes in the values of Sensory
Peak Latency and Motor onset latency between the two groups has not been significant as well.

Fateh manual therapy was safe, and no side effects were reported during the study.

Peripheral nerves during the passaging different regions of the body may be subjected to mechanical or chemical stimulation in different anatomical points. Long compression or fixation of a nerve may lead to reduction of blood flow in the nerves. ${ }^{37}$ This leads to the secretion of proinflammatory substances (peptides associated with Calcitonin gene and substance $\mathrm{P}$ ) of the nerve. This neurogenic inflammation can disrupt the normal function of the nerves even without obvious neurological damage, can also cause the creation and expansion of chronic pain. ${ }^{38}$

Researchers have shown that massage therapy is not only beneficial in the hands but also a significant reduction in pain scores in motor diseases, especially in fibromyalgia, waist pain, arthritis, and migraine..$^{12}$ And at the same time, studies have shown that massage has a role in reducing muscle tone and increasing local sympathetic activity in reducing pain intensity. ${ }^{12}$ The main clinical effects of manual therapy include reduced pain, improve performance, and neurological adjustment aspects. ${ }^{39}$

The response to nerve movement is complex and multifactor - physiological and psychological factors interfere with a complex method. Systematic studies have shown that moving nerves with multi-state care can improve symptoms, reduce disability and improve the function of patients who suffer from the peripheral nerve entrapment. It has been suggested that reducing inflammation and neural fibrosis after massage therapy can be the main reason for improving Functional Status and reducing Symptom Severity in patients with carpal tunnel syndrome. In carpal tunnel syndrome, in groups that received neural mobilization, positive neurophysiological effects such as intraocular edema reduction, time reduction, and median nerve shrinkage delay are observed..$^{40}$

There is increasing evidence that massage and joint manipulation adjust the mechanism of central pain. Massage activates descending inhibitory pathways using Oxytocin to produce analgesia. Manipulation reduces central irritability in individuals, as with a decrease in temporary accumulation in the primary hyperalgesia region and the reduction of secondary hyperalgesia in people with chronic pain. Therefore, manual therapy techniques activate central inhibitory mechanisms and reduce central irritability to develop analgesics in human and animal models. ${ }^{41}$

At the same time, the intervention of various types of manual therapy (for instance, massage therapy, physical 
therapy, chiropractic) prevents performance loss, improves task performance, preventing behavioral changes, which reduces nerve inflammation, degradation of myelin and Fibrosis extra nervous. ${ }^{37}$ In addition, passive traction may help reduce inflammation or intracranial pressure by stimulating the peripheral nerve as well as the associated vascular structures. ${ }^{42,43}$ These cases can justify a significant decrease in pain and improved Symptom Severity and Functional Status in the intervention group compared to the control group in this study.

\section{Limitations}

- Considering that so far there was not any study on this manual therapy method, therefore, in the present study, we compared it with a medical wristband and with only a group with simple treatment (splint only). For more detailed assessment, more experiments with stronger control groups (such as the use of massage simulation as a therapeutic method) as well as the use of the larger sample size are needed.

- Using different target scales to assess the severity of CTS symptoms, including VAS, BQ-SSS, BQ-FSS, and EDX parameters were our main strength points to compensate for some restrictions.

- In this study, despite the blinding of the electromyography process, filling the questionnaire, and the blinding of statistics analyzer, in order to receive manual therapy, there was no possibility of blinding patients.

- Although the base nerve and the tenth week electromyographies were performed by an expert for each patient, and despite the same EDX protocol, the electromyography of different patients was performed by two neurological and physical medicine specialists.

\section{Suggestions}

- In traditional Iranian medicine, a series of measures for treatment is used. Using manual therapy with chamomile oil (twice a day), which has been studied in the past (Setayesh et al., 2017), ${ }^{44}$ is among the measures that can lead to further improvement and more satisfaction in patients.

- The re-study with the greater number of patients, the comparison of the intervention group (solely with manual therapy) with two control groups (a group with a splint and a group with a splint and a pseudo-massage), as well as completing the full course for all participants is recommended to achieve more accurate results.

\section{Acknowledgment}

We thank all personnel of Shahid Modarres and Sina hospitals who helped us to implement this study. We also thank all the patients who kindly participate in this study.

\section{Declaration of Interests}

None.

\section{Funding}

This study was derived from the Ph.D. dissertation of Dr. Hamed Naeiji, School of Traditional Medicine, Shahid Beheshti University of Medical Sciences and the contract number for this study is 198 .

\section{References}

1. Lisa Newington, E.C.H., 2015. Carpal tunnel syndrome and work. Best Pract. Res. Clin. 29, 440-453.

2. Isam Atroshi Anna Jöud, Ingemar F. Petersson, Martin Englund, C.Z., 2015. Sickness Absence from Work among Persons with New PhysicianDiagnosed Carpal Tunnel Syndrome: A Population-Based Matched-Cohort Study 10.

3. Frederick M. Azar James H. Beaty, MD, S. Terry Canale, MD, M.D., 2017 Campbell's Operative Orthopaedics, Thirteenth. ed. Elsevier.

4. Agnessa Kozak Tanja Wirth, Ulrike Euler, Claudia Westermann and Albert Nienhaus, G.S., 2015. Association between work-related biomechanical risk factors and the occurrence of carpal tunnel syndrome: an overview of systematic reviews and a meta-analysis of current research. BMC Musculoskelet. Disord. 16. https://doi.org/10.1186/s12891-015-0685-0

5. Joseph Ingram Benjamin M. Mauck, MD,Norfleet B. Thompson, MD, James H. Calandruccio, MD, M.D., 2018. Cost, Value, and Patient Satisfaction in Carpal Tunnel Surgery. Orthop. Clin. North Am. 49. https://doi.org/doi. org/10.1016/j.ocl.2018.06.005

6. Tulipan, J.E., Ilyas, A.M., 2020. Carpal Tunnel Syndrome Surgery: What You Should Know. Plast Reconstr Surg Glob Open 8, e2692. https://doi. org/10.1097/gox.0000000000002692

7. Michel Chammas Lauren Marquardt Burmann, Renato Matta Ramos, Francisco Carlos dos Santos Neto, Jefferson Braga Silva, J.B., 2014. Carpal tunnel syndrome 49.

8. Parviz Yazdanpanah Ali Mousavizadeh, Ali Sahuli-Tanha, H.M., 2015. Incidence of Recurrent and Persistent Carpal Tunnel Syndrome following Open Transverse Carpal Ligament Release 17(1).

9. Prof. LucaPadua MD Carmen Erra MD, Costanza Pazzaglia MD, Ilaria Paolasso MD, Claudia Loreti BS, Pietro Caliandro MD, Lisa D Hobson-Webb MD, D.C.M.D., 2016. Carpal tunnel syndrome: clinical features, diagnosis, and management. Lancet Neurol. 15, 1273-1284. https://doi.org/https://doi. org/10.1016/S1474-4422(16)30231-9

10. Karimzadeh, A., Bagheri, S., Raeissadat, S.A., Bagheri, S., Rayegani, S.M., Rahimi-Dehgolan, S., Safdari, F., Abrishamkarzadeh, H., Shirzad, H., 2019. The comparison of the effectiveness between different doses of local methylprednisolone injection versus triamcinolone in Carpal Tunnel Syndrome: a double-blind clinical trial. J Pain Res 12, 579-584. https://doi. org/10.2147/jpr.S190652

11. H. Richard Winn, M.D., 2017. Neurological Surgery, Youmans. Elsevier.

12. Madenci, E., Altindag, O., Koca, I., Yilmaz, M., Gur, A., 2012. Reliability and efficacy of the new massage technique on the treatment in the patients with carpal tunnel syndrome. Rheumatol Int 32, 3171-3179. https://doi. org/10.1007/s00296-011-2149-7

13. Mohammad Hassan Bahrami Seyed Ahmad Raeissadat, S.S., 2015. Comparison between the effects of progesterone versus corticosteroid local injections in mild and moderate carpal tunnel syndrome: a randomized clinical trial 16.

14. Raeissadat SA Sedighipour L, Vahdatpour B, S.S., 2017. Randomized controlled trial of local progesterone vs corticosteroid injection for carpal tunnel syndrome.

15. Gary M. Franklin MPHa, Andrew S. Friedman, MD, M.D., 2015. Work-Related CarpalTunnel Syndrome, Diagnosis and Treatment Guideline. Phys. Med. Rehabil. Clin. N. Am. 26. https://doi.org/http://dx.doi.org/10.1016/j. pmr.2015.04.003 
16. Rayegani SM Eliaspour D, Raeissadat SA, Shafi Tabar Samakoosh M, Sedihgipour L, Kargozar E, B.M.H., 2013. The effects of low intensity laser on clinical and electrophysiological parameters of carpal tunnel syndrome 4.

17. Yi Huey Lim BSc Sonya Girdler PhD, Hoe C. Lee PhD., D.Y.C.Bs., 2017. Median nerve mobilization techniques in the treatment of carpal tunnel syndrome: A systematic review. J. Hand Ther. 30. https://doi.org/http://dx.doi. org/10.1016/j.jht.2017.06.019

18. Dinarvand, V., Abdollahi, I., Raeissadat, S.A., Mohseni Bandpei, M.A., Babaee, M., Talimkhani, A., 2017. The Effect of Scaphoid and Hamate Mobilization on Treatment of Patients with Carpal Tunnel Syndrome. Anesth Pain Med 7, e14621. https://doi.org/10.5812/aapm.14621

19. Raeissadat SA Rezaei S, Sedighipour L, Bahrami MH, Eliaspour D, Karimzadeh A, R.S.A., 2014. The Effect of Polarized Polychromatic Noncoherent Light (Bioptron) Therapy on Patients with Carpal Tunnel Syndrome 5.

20. Carlson, H., Colbert, A., Frydl, J., Arnall, E., Elliot, M., Carlson, N., 2010. Current options for nonsurgical management of carpal tunnel syndrome. Int J Clin Rheumtol 5, 129-142. https://doi.org/10.2217/ijr.09.63

21. Raeissadat, S.A., Karimzadeh, A., Hashemi, M., Bagherzadeh, L., 2018. Safety and efficacy of platelet-rich plasma in treatment of carpal tunnel syndrome; a randomized controlled trial. BMC Musculoskelet Disord 19, 49. https://doi. org/10.1186/s12891-018-1963-4

22. Bahrami, M.H., Raeissadat, S.A., Nezamabadi, M., Hojjati, F., RahimiDehgolan, S., 2019. Interesting effectiveness of ozone injection for carpal tunnel syndrome treatment: a randomized controlled trial. Orthop Res Rev 11, 61-67. https://doi.org/10.2147/orr.S202780

23. Wolny, T., Saulicz, E., Linek, P., Shacklock, M., Myśliwiec, A., 2017. Efficacy of Manual Therapy Including Neurodynamic Techniques for the Treatment of Carpal Tunnel Syndrome: A Randomized Controlled Trial. J Manip. Physiol Ther 40, 263-272. https://doi.org/10.1016/j.jmpt.2017.02.004

24. Wolny, T., Linek, P., 2019a. Long-term patient observation after conservative treatment of carpal tunnel syndrome: a summary of two randomised controlled trials. PeerJ 7, e8012. https://doi.org/10.7717/peerj.8012

25. Afrasiabian H Shams Ardakani MR, F.G.A., 2015. Essentials of Ghamz in Iranian Traditional Medicine (Mabany-e-Ghamz dar Tebb-e-Sonnatiy-eIran). Nameh Hasti, Tehran.

26. Foroozanfar, Z., Ebrahimi, H., Khanjani, N., 2015. Validity and Reliability of the Persian Boston Questionnaire in Diabetic Patients with Carpal Tunnel Syndrome. J. Neyshabur Univ. Med. Sci. 2, 50-56.

27. Rezazadeh, A., Bakhtiary, A.H., Samaei, A., Moghimi, J., 2014. Validity and reliability of the Persian Boston questionnaire in Iranian patients with carpal tunnel syndrome. koomesh J. 15, 138-145.

28. Sanei, M., Roozafzai, F., Abousaidi, S.R., Hamze, M., Negarestani, A.M., Mokaberinejad, R., 2020. Persian manual therapy method for chronic low-back pain with lumbar radiculopathy; a randomized controlled trial. J Bodyw Mov Ther 24, 123-130. https://doi.org/10.1016/j.jbmt.2020.02.015

29. Elliott, R., Burkett, B., 2013. Massage therapy as an effective treatment for carpal tunnel syndrome. J Bodyw Mov Ther 17, 332-338. https://doi org/10.1016/j.jbmt.2012.12.003

30. Jaladat, A.M., Atarzadeh, F., Homayouni, K., 2013. Ghamz therapy in Persian Medicine and its comparison with Reflexo Zone therapy. J. Islam. Iran. Tradit. Med. 3, 395-406.

31. Mohammadi-Kenari, H., Khoramizadeh, M., Forogh, B., Hashem-Dabaghian, F., 2020. The Effect of a Persian Medicine Massage (Dalk and Ghamz) on Neck Pain and Disability in Nonspecific Chronic Neck Pain. Complement. Med. Res. https://doi.org/10.1159/000509051

32. Fernández-de-Las Peñas, C., Ortega-Santiago, R., de la Llave-Rincón, A.I. Martínez-Perez, A., Fahandezh-Saddi Díaz, H., Martínez-Martín, J., Pareja,
J.A., Cuadrado-Pérez, M.L., 2015. Manual Physical Therapy Versus Surgery for Carpal Tunnel Syndrome: A Randomized Parallel-Group Trial. J Pain 16, 1087-1094. https://doi.org/10.1016/j.jpain.2015.07.012

33. Moraska, A., Chandler, C., Edmiston-Schaetzel, A., Franklin, G., Calenda, E.L, Enebo, B., 2008. Comparison of a targeted and general massage protocol on strength, function, and symptoms associated with carpal tunnel syndrome: a randomized pilot study. J Altern Complement Med 14, 259-267. https:// doi.org/10.1089/acm.2007.0647

34. Lewis, K.J., Coppieters, M.W., Ross, L., Hughes, I., Vicenzino, B., Schmid, A.B., 2020. Group education, night splinting and home exercises reduce conversion to surgery for carpal tunnel syndrome: a multicentre randomised trial. J Physiother 66, 97-104. https://doi.org/10.1016/j. jphys.2020.03.007

35. Maddali Bongi, S., Signorini, M., Bassetti, M., Del Rosso, A., Orlandi, M., De Scisciolo, G., 2013. A manual therapy intervention improves symptoms in patients with carpal tunnel syndrome: a pilot study. Rheumatol Int 33 1233-1241. https://doi.org/10.1007/s00296-012-2507-0

36. Wolny, T., Linek, P., 2019b. Is manual therapy based on neurodynamic techniques effective in the treatment of carpal tunnel syndrome? A randomized controlled trial. Clin Rehabil 33, 408-417. https://doi. org/10.1177/0269215518805213

37. Bove, G.M., Delany, S.P., Hobson, L., Cruz, G.E., Harris, M.Y., Amin, M., Chapelle, S.L., Barbe, M.F., 2019. Manual therapy prevents onset of nociceptor activity, sensorimotor dysfunction, and neural fibrosis induced by a volitional repetitive task. Pain 160,632-644. https://doi.org/10.1097/j. pain.0000000000001443

38. Matsuda, M., Huh, Y., Ji, R.R., 2019. Roles of inflammation, neurogenic inflammation, and neuroinflammation in pain. J Anesth 33, 131-139. https://doi.org/10.1007/s00540-018-2579-4

39. Salgado, A.S.I., Stramosk, J., Ludtke, D.D., Kuci, A.C.C., Salm, D.C., Ceci, L.A., Petronilho, F., Florentino, D., Danielski, L.G., Gassenferth, A., Souza, L.R., Rezin, G.T., Santos, A.R.S., Mazzardo-Martins, L., Reed, W.R., Martins, D.F., 2019. Manual Therapy Reduces Pain Behavior and Oxidative Stress in a Murine Model of Complex Regional Pain Syndrome Type I. Brain Sci 9. https://doi. org/10.3390/brainsci9080197

40. Basson, A., Olivier, B., Ellis, R., Coppieters, M., Stewart, A., Mudzi, W., 2017. The Effectiveness of Neural Mobilization for Neuromusculoskeletal Conditions: A Systematic Review and Meta-analysis. J Orthop Sport. Phys Ther 47 593-615. https://doi.org/10.2519/jospt.2017.7117

41. Chimenti, R.L., Frey-Law, L.A., Sluka, K.A., 2018. A Mechanism-Based Approach to Physical Therapist Management of Pain. Phys Ther 98, 302-314. https://doi.org/10.1093/ptj/pzy030

42. Boudier-Revéret, M., Gilbert, K.K., Allégue, D.R., Moussadyk, M., Brismée, J.M., Sizer Jr., P.S., Feipel, V., Dugailly, P.M., Sobczak, S., 2017. Effect of neurodynamic mobilization on fluid dispersion in median nerve at the level of the carpal tunnel: A cadaveric study. Musculoskelet Sci Pr. 31, 45-51. https://doi.org/10.1016/j.msksp.2017.07.004

43. Gilbert, K.K., Smith, M.P., Sobczak, S., James, C.R., Sizer, P.S., Brismée, J.M., 2015. Effects of lower limb neurodynamic mobilization on intraneural fluid dispersion of the fourth lumbar nerve root: an unembalmed cadaveric investigation. J Man Manip Ther 23, 239-245. https://doi.org/10.1179/2042 618615y.0000000009

44. Setayesh, M., Sadeghifar, A.R., Nakhaee, N., Kamalinejad, M., Rezaeizadeh, H., 2017. A Topical Gel From Flax Seed Oil Compared With Hand Splint in Carpal Tunnel Syndrome: A Randomized Clinical Trial. J Evid Based Complement. Altern Med 22, 462-467. https://doi. org/10.1177/2156587216677822 